\title{
Winning hearts and minds: una mirada ética desde la antropología a la Acción Integral en Colombia ${ }^{1}$
}

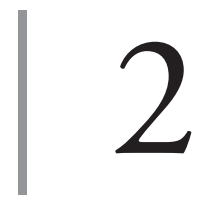

\author{
https://doi.org/10.21830/9789585377141.02
}

Eva Maria Rey Pinto ${ }^{2}$ Angélica María González González ${ }^{3}$ Escuela Superior de Guerra "General Rafael Reyes Prieto" Juan David Mora Peña ${ }^{4}$ Universidad Militar Nueva Granada

\section{Resumen}

El presente capítulo tiene como objetivo realizar un análisis, desde la antropología, sobre los retos éticos que se le presentan a la Acción Integral en Colombia, específicamente en el escenario de posacuerdo actual. Esto se realizó a través de una metodología cualitativa de carácter comparativo, la cual permitió caracterizar el caso de estudio de la Acción Integral en Colombia y el Human Terrain System en Estados Unidos. Se identificó que los

1 Este capítulo presenta los resultados colaborativos de dos proyectos de investigación: (1) "Crimen Organizado Transnacional: dimensiones culturales y capacidades", del Centro Regional de Estudios Estratégicos en Seguridad (CREEs), vinculado al grupo de investigación Masa Crítica, de la Escuela Superior de Guerra "General Rafael Reyes Prieto", Colombia, categorizado en B por Minciencias y con código de registro COL0123247, y (2) "Mujeres de arma, seguridad y defensa nacional. Un análisis desde sus percepciones”, del grupo de investigación en Ciencias Militares, de la Escuela Militar de Cadetes "General José María Córdova", Colombia, categorizado en B por Minciencias y con código de registro COL0082556. Los puntos de vista pertenecen a los autores y no reflejan necesariamente los de las instituciones participantes.

2 Magíster en Estrategia y Geopolítica de la Escuela Superior de Guerra "General Rafael Reyes Prieto". Antropóloga de la Universidad de los Andes. Investigadora del Centro Regional de Estudios Estratégicos en Seguridad (CREes) y asistente editorial de la Revista Cientifica Estudios en Seguridad y Defensa. OrCID: https://orcid.org/0000-0003-2004-7466 - Contacto: eva.rey@esdegue.edu.co

3 Candidata a magíster en Estrategia y Geopolítica de la Escuela Superior de Guerra "General Rafael Reyes Prieto”. Politóloga con énfasis en Estudios de Seguridad, Paz y Conflicto de la Universidad del Rosario. Orcid: https://orcid.org/0000-0003-0881-5530 - Contacto: gonzalezam@esdegue.edu.co

4 Magíster en Estrategia y Geopolítica de la Escuela Superior de Guerra "General Rafael Reyes Prieto”. Especialista en Estudios Políticos de la Universidad Sergio Arboleda. Abogado de la Pontificia Universidad Javeriana. Investigador en el Instituto de Estudios Geoestratégicos y Asuntos Políticos de la Universidad Militar Nueva Granada. ORCID: https://orcid.org/0000-0002-2952-9608 - Contacto: juand. mora@unimilitar.edu.co 
principales retos, desde una mirada ética, a la Acción Integral en Colombia, y con base en las lecciones aprendidas del caso norteamericano, son: el manejo y uso de la información, la configuración del otro como enemigo y el rol de las ciencias sociales en la lucha contrainsurgente. Se concluye que es necesario reflexionar sobre los dilemas éticos que supone la construcción de estrategias como la Acción Integral, con el fin de que puedan contribuir de manera correcta a la construcción de paz en el escenario de posacuerdo.

Palabras clave: Acción Integral; antropología; Colombia; ética; Human Terrain System.

\section{Introducción}

La Acción Integral (AIN) en Colombia es una de las apuestas de las Fuerzas Militares que ha tenido un desarrollo amplio a través del tiempo, de manera que se ha enfrentado a nuevos retos en los medios donde participa. Hoy en día la Ain está en el marco de un escenario de posacuerdo y uno de sus principales desafíos es la construcción de paz de la mano de otros actores. Sin embargo, esta estrategia, al ejecutar operaciones psicológicas, como parte de su planteamiento, debe tener una mirada crítica hacia el desarrollo de sus prácticas. Por esto, este capítulo se centra en explorar los retos de la AIN como herramienta de construcción de paz, a través de los antecedentes de las nuevas formas de guerra, un estudio comparado que evidencia una serie de elementos que se deben tener en cuenta para ejercer una práctica responsable y una contextualización propia de la AIN en Colombia, que explica los retos de la construcción de paz por este medio.

Así, en la primera parte del trabajo se hace un análisis de las perspectivas de combate que han traído consigo las nuevas guerras posteriores a la Guerra Fría y la subsecuente aparición de las operaciones militares distintas a la guerra. Entre estas se encuentran las operaciones psicológicas y las operaciones cívico-militares, como consecuencia de sucesos históricos que han hecho necesario que las Fuerzas Militares trabajen con la población y la información. Así, se presenta una breve visión de la AIN en Colombia desde su surgimiento como estrategia de lucha contrainsurgente y sus objetivos principales.

La segunda parte explora cómo desde la antropología se ha entendido el impacto de las operaciones psicológicas, al establecer una serie de observa- 
ciones a partir de la experiencia del Human Terrain System (HTS), la cual abre un debate importante en el uso de la cultura como arma de guerra, y que tiene una variedad de similitudes con la AIN. Esta sección discute así las prácticas de ambos programas, los dilemas éticos existentes y el papel de las responsabilidades institucionales. Con esto se busca reconocer la necesidad de aprender las lecciones que dejó dicha experiencia de las Fuerzas Militares norteamericanas y continuar creando conciencia sobre el desarrollo de la AIN en los territorios golpeados por la guerra en Colombia en el escenario del posacuerdo.

La tercera parte busca comprender el entorno en el cual se desarrolla actualmente la doctrina de AIN en Colombia, para lo cual se analiza una serie de factores que la transformaron y enfocaron en términos de nuevas nociones de seguridad, el contexto de las relaciones civiles-militares, las acciones cívico-militares, los nuevos roles que deben desarrollar las Fuerzas Militares, y la aplicación de la AIN para la consolidación territorial y de los gobiernos regionales y locales, en el marco del posacuerdo de paz con la guerrilla de las FARC firmado el 26 de septiembre del 2016, para determinar cómo esta herramienta es importante en la construcción de paz. En ese sentido, se realiza un análisis histórico de los factores mencionados para comprender las nuevas amenazas a las que se debe enfrentar la doctrina.

Finalmente, las tres partes expuestas se conjugan en unas reflexiones finales, donde se expone la importancia de los tres argumentos desarrollados para la construcción de paz en Colombia, a través de las funciones de la AIN en la consolidación de los territorios, dentro de un marco ético aprendido por medio de las lecciones que dejan experiencias anteriores.

\section{Las operaciones militares distintas a la guerra como modos de combate}

La guerra, como gran parte de las actividades humanas, ha avanzado en diferentes etapas a lo largo de la historia, de tal manera que las nuevas perspectivas de combate han obligado a los actores militares a estructurar nuevas tácticas que respondan eficientemente en la búsqueda de los objetivos operacionales. Para Carl von Clausewitz (1999), la guerra es un método único de 
los Estados para alcanzar sus intereses políticos frente a sus contrarios, de tal modo que se constituye como una herramienta esencial para su supervivencia y expansión. En tal consideración, el conflicto armado pretende ser un instrumento restringido siempre en la idea de la derrota de la voluntad del enemigo, quien desiste en sus intereses políticos a favor del vencedor. No obstante, a pesar de lo sistemática que pueda resultar la idea de la guerra de Clausewitz, es evidente que esta esconde elementos más allá de lo militar que se deben tener en consideración, tal como lo expone Paret (1992) en el capítulo introductorio de su obra Creadores de la estrategia moderna, al decir:

Todos reconocen que la guerra no ha sido nunca, ni es hoy en día, un fenómeno básicamente militar, ni tampoco solamente militar, sino que es la combinación de muchos elementos que abarcan desde la política y la tecnología, a las emociones humanas sometidas a tensiones extremas, es decir, la guerra, sobre todo en la actualidad, ya no responde a la acción meramente militar, sino que abarca una multiplicidad. (p. 20)

Para la profesora Mary Kaldor (2012), la guerra ha obtenido unas nuevas características que la diferencian con respecto a la idea clásica de Clausewitz, entre las cuales se evidencia la utilización de nuevas maneras de combate y la proliferación de actores militares aparte de los Estados. Estos nuevos elementos han impactado la población civil, ya que "hay un desdibujamiento de límites en la guerra, adquiriendo importancia la presencia del crimen organizado ejercido por grupos privados y las violaciones a gran escala de los Derechos Humanos, no solo por los Estados, sino también por grupos organizados políticamente" (Bados \& Durán, 2015 p. 12). Ahora, para Kaldor (2012), el concepto de Frank Hoffman de guerra híbrida expresa de forma correcta los nuevos modos de combate que son usados en la actualidad.

El Teniente Coronel de los Estados Unidos, Frank Hoffman (2007), resalta el carácter de amplitud y la nueva búsqueda por el elemento psicológico de la población civil en las guerras híbridas.

Las amenazas híbridas incorporan un amplio rango de modos de combate, incluyendo capacidades de tipo convencional, tácticas y formaciones irregulares, actos terroristas incluyendo violencia y coerción indiscriminada y desorden criminal [...] que coordinadas con el principal terreno de batalla 
alcanzarán efectos sinergéticos en las dimensiones físicas y psicológicas del conflicto (p. 8)

Por lo anterior, el Coronel Rodolfo Ortega (2011), del Ejército de Chile, advierte que posterior a Vietnam existió un cambio importante en la mentalidad militar dentro de los cuerpos castrenses de los Estados Unidos, quienes han desarrollado en la actualidad las categorías de Operaciones Militares de Guerra, como aquellas actividades de uso de la fuerza militar convencional, y las Operaciones Militares Distintas a la Guerra (OPDG), enfocadas en "disuadir en el uso de la fuerza, promover la paz, el orden y apoyo a la ciudadanía" (Ortega, 2011, p. 26). Según el autor, las OPdG contienen un abanico importante de actividades que pueden impactar en las operaciones de combate y en la conducción de la guerra. Entre estas se cuentan las operaciones "de paz, humanitarias, de búsqueda y rescate, evacuación de no combatientes, apoyo militar a autoridades civiles, implantación de sanciones o embargos, vigilancia y protección de fronteras o aplicación de la ley" (Ortega, 2011, p. 26), así como las que llama Operaciones de Información, dentro de las cuales se enmarcan las bien conocidas operaciones psicológicas. Este nuevo tipo de operaciones militares ha reforzado la necesidad de diversificar el esfuerzo militar y de ampliar el rango de actividades de las Fuerzas Militares de la actualidad, sobre todo en la relación de las tropas con la población civil en las denominadas acciones cívico-militares.

Ahora, es evidente que las OPDG no son únicas de la modernidad, pues el uso de la información y la voluntad de la población civil con objetos militares se han visto desde los escenarios más antiguos de la guerra. Sun Tzu, estratega chino, consideraba que la mejor forma de lograr una victoria era sin luchar: "De este modo los expertos en la guerra someten un ejército enemigo sin librar combates. Capturan sus ciudades sin asedios y desbaratan su gobierno sin largas operaciones" (Sun Tzu, 1999, p. 120), planteamiento que se puede someter a la consideración de Clausewitz (1999), quien expone que la victoria militar se logra únicamente sometiendo la voluntad de lucha del enemigo y no, simplemente, con la destrucción de sus fuerzas militares y la conquista del territorio. Esto fue implementado por Gengis Khan, emperador de los mongoles y creador del mayor imperio continuo de la historia, quien, gracias 
al manejo de la información acerca de la brutalidad de sus ejércitos, lograba que importantes ciudades se rindieran al ver su ejército cabalgando hacia sus murallas (Goldstein \& Jocobowitz, 1996).

De la misma manera, al iniciar la Primera Guerra Mundial, los países en pugna encontraron en la propaganda de guerra un método efectivo para derrotar la voluntad del enemigo e incentivar el fervor patriótico de sus propios ciudadanos, acto que fue reforzado y especializado durante la Segunda Guerra Mundial y la Guerra Fría (Goldstein \& Jocobowitz, 1996). En la actualidad, este tipo de OPDG, llamado ahora operaciones psicológicas, busca un acercamiento a la información más agresivo, de manera que el control de los medios de comunicación, la difusión de información beneficiosa y el manejo de las fuentes son elementos clave para la conducción de la guerra (Lind \& Thiele, 2015). Por tanto, para Lind y Thiele (2015), la prensa resulta ser una herramienta clave para el desarrollo de las operaciones militares, tanto para la desmoralización del enemigo como para aumentar el apoyo de la población civil.

Por otro lado, la relación de las fuerzas militares con la población, como OPDG, responde de igual manera a alcanzar los fines de combate. El objetivo reviste en integrar a la población civil en el esfuerzo de guerra con el fin de evitar actividades de inteligencia o de engrosar las tropas del enemigo. Para Lind y Thiele (2015), este es uno de los mayores errores cometidos por las Fuerzas Militares de los Estados Unidos en sus operaciones en Oriente Medio, ya que se olvida que "en vez de hacer a las fuerzas del Estado menos segura, la integración mejorará su seguridad a largo plazo. La razón es que si la tropa protege a los locales, los locales los protegerán a ellos" (p. 49).

El Teniente General David Petraeus (2006), comandante de la MultiNational Security Transition Command en Irak, expone como recomendaciones elementos que buscan, no solo lograr capturar las mentes y los corazones de los iraquíes, sino también el involucramiento de las Fuerzas Militares de los Estados Unidos, no como fuerza invasora, sino como una fuerza de liberación. Por tanto, Petraeus (2006) muestra la importancia de incentivar la readecuación de los daños causados por las guerras de forma rápida, el involucramiento de las entidades gubernamentales locales en la reestructuración de un 
nuevo país, la recuperación de instituciones que impulsen el orgullo nacional dañado, entre otras actividades. Todo esto para evitar el apoyo de la población al enemigo y la aversión a la fuerza americana.

Por otro lado, a pesar del debate en torno al posicionamiento del conflicto armado en Colombia con respecto a las teorías de las nuevas guerras de Kaldor “(El Espectador, 2017)”. el Estado colombiano no ha sido ajeno a los avances militares respecto a las OPDG en los nuevos tipos de combate. En el año 1959, habiendo pasado eventos tan importantes para el Ejército colombiano como la Guerra de Corea o el gobierno militar del Teniente General Gustavo Rojas Pinilla, el General Álvaro Valencia Tovar (1959) describe la guerra diciendo que "ha dejado de ser la destrucción material significada en la muerte física de millones de hombres sobre un desgarrado campo de batalla o en la visión dantesca de ciudades arrasadas para convertirse en la muerte moral de las conciencias" (p. 396). De esta manera, Valencia Tovar (1959) entendía que la guerra revolucionaria revestía un complejo elemento que impedía que los ejércitos regulares pudieran luchar abiertamente, es decir, la guerra total por todos los medios.

A raíz de estos esfuerzos realizados por veteranos de la Guerra de Corea como el General Valencia Tovar y con el fin de luchar efectivamente en contra de los métodos propagandísticos de las guerrillas colombianas, se creó el Plan Laso en 1964. Este plan, tras un análisis de las razones del conflicto, encuentra que la guerra de tipo revolucionario que evoca las disposiciones de Mao Tse Tung y el Che Guevara, entre otros, buscan en la población civil un insumo más para alcanzar una base social y posteriormente una acción militar de tipo convencional (Niño \& Castillo, 2018), por tal motivo, el Ejército encontró la extrema necesidad de tener a la población civil, sobre todo al campesinado, de su lado.

Con el tiempo, el impulso iniciado por el Plan Laso, después complementado por el Plan Perla y el Plan Andes, dio paso a la consolidación de una estrategia interinstitucional para la realización de Operaciones de Información (incluidas las operaciones psicológicas) y las operaciones cívico-militares, la cual sería recuperada por la Política de Seguridad Democrática del presidente Álvaro Uribe Vélez (Valencia, 2006). 
La AIN, por tanto, es definida por el General Mario Montoya (2007) como un

compendio de factores o elementos articulados y coordinados para fortalecer las estructuras institucionales del Estado, en procura del bienestar de la colectividad, fundamentado en la legitimidad, legalidad, el respeto a la dignidad humana, la protección y difusión de los Derechos Humanos para todos los ciudadanos. (p. 21)

Por lo tanto, la AIN se constituye como un mecanismo para luchar contra el enemigo y "causarle el mayor daño posible, deslegitimándolo, mediante el máximo empleo de la propaganda, la desinformación y otras acciones de orden político, socioeconómico, religioso, cultural y militar” (Montoya, 2007, p. 21), para así alcanzar la victoria militar.

\section{Relaciones civiles-militares en el marco de la Acción Integral y los procesos de paz}

Para comprender cómo se ha desarrollado la doctrina de Acción Integral en términos de relaciones civiles-militares es necesario comprender que, si bien esta doctrina se da desde el gobierno del General Gustavo Rojas Pinilla (1953-1957), las relaciones entre la sociedad civil y las Fuerzas Militares se evidencian desde la Independencia de Colombia y se presentan como un fenómeno tan distinto a los demás países de la región que amerita ser estudiado de manera puntual.

Desde el proceso de Independencia (1810-1819) hasta la creación de los partidos políticos en 1849, en Colombia se establece una clara tendencia y vocación civil donde los partidos políticos y las élites sociales han sido hábiles para movilizar los recursos del Estado, de tal manera que se han convertido en el eje principal de la vida política y económica, con lo cual se relega a los ejércitos y crece un sentimiento casi antimilitarista que se vio reflejado en el constante rechazo a la profesionalización militar. Ya entrando al siglo XX, se puede observar un fenómeno tardío de profesionalización en las Fuerzas para la instrumentalización de los ejércitos por parte de los partidos políticos, y con 
el establecimiento del Frente Nacional aparece la figura de estado de sitio, con lo cual el gobierno reduce la posición de los ejércitos al modelo de mantenimiento del orden público (Moreno, 2014).

Este modelo generó tensiones en los gobiernos posteriores de Belisario Betancur (1982-1986) cuando se iniciaron las negociaciones de paz con las insurgencias de las FARC, el EPL, el M-19 y el ELN. En este contexto se observó un mensaje de división entre las esferas política y militar, que dio relevancia a la esfera civil y que terminó con la toma del Palacio de Justicia, cuando Betancur afirmó que la responsabilidad del ingreso de tanques de guerra era del Ejército y su comandante general. Posteriormente, con el gobierno de Virgilio Barco (1986-1990), cuando se logró el proceso de paz con el M-19, se introdujeron civiles en cargos relacionados con temas de seguridad, lo cual llevó a que en el gobierno de César Gaviria (1990-1994) se designara por primera vez a un civil como ministro de Defensa y se creara en 1991 la Escuela de Relaciones Civiles y Militares del Ejército (ESREM), donde se estableció la doctrina de cooperación cívico-militar y se buscó una nueva capacitación para los miembros del Ejército, la Armada y la Fuerza Aérea en operaciones psicológicas (Leal, 1994).

Tiempo después, con la llegada de Álvaro Uribe Vélez (2002-2010) al poder, después del proceso de paz fallido del gobierno de Andrés Pastrana (1998-2002), se generó una subordinación desinstitucionalizada y se reactivó la Doctrina de Seguridad Nacional (DSN), con la cual se amplió la autonomía de las Fuerzas Militares y se encuadró con la política de la guerra contra el terrorismo promovida por los Estados Unidos. De esta manera, se llevó a cabo la actualización de la doctrina contrainsurgente del enemigo interno de la DSN, que reemplazó la categoría de insurgencias por el terrorismo. Esta política pretendió unir a los ciudadanos con las autoridades poniendo a la población al servicio de las Fuerzas Militares en programas como Soldados Campesinos y Redes de Informantes, sin embargo, esta interacción no permitía que los ciudadanos participaran en los procesos de formulación de políticas (Cruz, 2015).

En el marco de la doctrina AIN, con la llegada de Álvaro Uribe Vélez se presenta la Política de Seguridad Democrática (PSD), en la cual la doctrina se adopta como estrategia para la recuperación del territorio. Puntualmente, 
esta política estaba enmarcada en el "restablecimiento del imperio de la ley, la garantía del Estado social de derecho, el libre ejercicio de DD. HH. y la promoción de los fines del Estado", con lo cual de 2007 a 2010 se fijó la AIN como responsable de preparar las condiciones de bienestar social y neutralización de las amenazas de grupos armados ilegales. Es por esta razón que en 2007 se realizó el Plan de Consolidación Integral de La Macarena (РСIM) para la erradicación de cultivos ilícitos, la reducción de los índices de violencia y el desplazamiento forzado ofreciendo cobertura a las necesidades básicas y educación básica (Silva, 2014).

Con la llegada a la presidencia de Juan Manuel Santos se presentan una serie de retos y oportunidades frente al proceso de paz, debido a que las Fuerzas Militares se transformaron en su tamaño, capacidad y organización, comparado con la negociación del gobierno Pastrana, que se estableció tras una serie de derrotas militares. La estructura de las Fuerzas Militares en la presidencia de Juan Manuel Santos se establecía tras importantes golpes a estructuras guerrilleras y un crecimiento en tamaño y recursos (Illera \& Ruiz, 2018). Posteriormente, con la aparición de la Política Integral de Seguridad y Defensa Nacional del gobierno Santos, la AIN se estableció como un eje trascendental reservado a recuperar la confianza de la sociedad civil accediendo a zonas históricamente golpeadas por el conflicto armado para resolver las necesidades de la población más vulnerable en torno a metas de corto, mediano y largo plazo (Rodríguez, 2014).

A corto plazo se busca la ejecución de proyectos de infraestructura desde la Jefatura de Ingenieros Militares para la construcción de vías que conectan municipios aislados y la edificación de comedores comunitarios, además de jornadas de salud y bienestar. A mediano plazo, se busca aumentar los grupos de apoyo a las unidades de AIN para la construcción de una relación con las comunidades étnicas que permita el libre acceso a sus territorios y disuada a los grupos al margen de la ley que se encuentren en sus territorios. A largo plazo se proyecta la etapa de posconflicto, con lo cual se busca realizar proyectos de infraestructura permanente, en términos de atención a desastres, desminado humanitario y obras civiles en las zonas más distantes y complejas del territorio nacional (Rodríguez, 2014). 
A partir de lo anterior, se puede resumir que la relación civico-militar en el marco de la doctrina de AIN sirve para conseguir el respaldo de la población a las Fuerzas Militares desde la teoría de la cooperación cívico-militar conocida como acción cívico-militar en el marco de la acción unificada. De esta manera, se sincronizan y coordinan las actividades entre el gobierno, el sector privado, la sociedad civil organizada, la cooperación internacional y formas de participación comunitaria, además de que se contribuye al mejoramiento de la calidad de vida de la comunidad y se busca el rechazo de la población frente a los actores al margen de la ley (Ruiz, 2007).

En ese sentido, la AIN se perfila como un mecanismo de lucha que ha evolucionado dentro de las Fuerzas Militares de Colombia para alcanzar la victoria militar frente a las nuevas amenazas que proponen los modos de combate modernos y los actores de las nuevas guerras conceptualizadas por Mary Kaldor. También cabe mencionar que la evolución de la AIN en Colombia ha sido permeada por las relaciones militares con países aliados, sobre todo de los Estados Unidos, pero también ha tenido un cariz único surgido de los conocimientos de generales, oficiales y demás miembros de las Fuerzas Militares y las ciencias sociales desde la academia.

\section{Human Terrain System y Acción Integral: prácticas y dilemas éticos}

Las operaciones contrainsurgentes han representado un reto para las instituciones militares debido a que las insurgencias poseen conocimientos locales que les significan ventajas importantes (McFate \& Jackson, 2006). Como se evidenció anteriormente, las operaciones militares han tenido que adaptarse a este tipo de retos a través del tiempo, por medio de una variedad de programas que han permitido desarrollar las denominadas operaciones psicológicas, que han sido, en su diversidad, las principales estrategias para la guerra contrainsurgente. Este tipo de operaciones tienen un enfoque particular en la necesidad de entender al "enemigo" — categoría que se problematiza más adelante- a través de sus prácticas sociales y culturales. Con base en una de las principales premisas de la guerra, "conoce a tu enemigo", se han llevado a cabo no solo 
enfrentamientos directos, sino otro tipo de prácticas imperialistas que desde el siglo XIX han implementado herramientas, conocimientos y recursos humanos dedicados a las ciencias sociales, especialmente a la antropología, la cual "fue desarrollada, en gran parte, para apoyar la empresa militar. Frecuentemente llamada 'la doncella del colonialismo', el conocimiento antropológico contribuyó a la expansión y consolidación del poder británico durante la era del imperio" (McFate, 2005, p. 47).

Precisamente, por la necesidad de conocer al otro, que se construye como enemigo, la antropología resultó ser una disciplina de interés para las instituciones militares. Uno de los antecedentes que marcó esta práctica fueron los esfuerzos contrainsurgentes en la Guerra de Vietnam, que se denominaron el Civil Operations Revolutionary Development Support (CORDS), una estrategia para realizar inteligencia por medio de la implementación del Programa Phoenix, que resultó ser un fracaso porque la información recopilada fue manipulada para asesinar de manera indiscriminada (González, 2008). Si bien este tipo de operaciones que se dedicaban a la búsqueda y la recopilación de información ha tenido un enfoque en entender las culturas, no se estableció una práctica sistemática de la mano de la antropología hasta el siglo XXI después del 9/11. Esta práctica se denominó el Human Terrain System (HTs) y ha sido uno de los experimentos más polémicos llevados a cabo por las Fuerzas Militares norteamericanas, el cual se desarrolló entre 2006 y 2014. Debido a la similitud de algunos componentes de esta práctica con la formulación y el desarrollo de la AIN en Colombia, es necesario hacer un ejercicio de reflexión sobre las lecciones que dejó el HTs y los dilemas éticos a los que se enfrentaron las instituciones militares.

El HTs fue un programa implementado por las Fuerzas Militares estadounidenses que tenía como fin desplegar científicos sociales en Irak y Afganistán, previamente entrenados en combate y junto con militares activos, para realizar operaciones psicológicas que debían, a través de la recopilación de información, identificar amenazas y potenciales enemigos a través de la introspección cultural. De hecho, la expresión Human Terrain se define como "los elementos sociales, etnográficos, culturales, económicos y políticos de las personas, entre las que opera una fuerza, definidos y caracte- 
rizados por datos socioculturales, antropológicos y etnográficos" (Kipp et al., 2006, p. 9; citado por González, 2008, p. 22); como se muestra, esta expresión tiene como protagonistas al trabajo etnográfico y a la antropología. Así, al momento de crear todo un sistema alrededor del HTs fue clave el asesoramiento de la antropóloga Montgomery McFate, quien tenía experiencia en la formulación de política de seguridad y defensa, además de que ya había realizado artículos reflexionando sobre la necesidad de "antropologizar" la lucha contrainsurgente (McFate, 2005).

Así, entre los años 2005 y 2006 se construyó el HTs, el cual se presentó al inicio como el programa estrella que permitiría disminuir las operaciones hostiles en terreno, salvando vidas a través de operaciones asesoradas por personal civil capacitado en áreas como la antropología, la sociología, la ciencia política y la economía. Al comienzo, gracias a una campaña publicitaria en medios, el programa tuvo una gran acogida, pero con el paso del tiempo se fue evidenciando una serie de problemas, los cuales provocaron, después de diversos debates en ámbitos políticos y académicos, la cancelación total del programa en 2014, sin evidencia de resultados satisfactorios. Para los objetivos de este trabajo, las críticas que enfrentó este programa se dividirán en tres: en primer lugar, el uso de herramientas y conocimientos de las ciencias sociales; segundo, el manejo de la información y, tercero, los dilemas éticos de la práctica. Estos elementos serán explicados a través del HTs y, a su vez, contrastados con los principios de la AIN en Colombia, con el fin de reflexionar sobre la necesidad de establecer unas prácticas institucionales responsables que conduzcan a la construcción de paz.

\section{¿Ciencias sociales al servicio de la lucha contrainsurgente?}

Anteriormente se expuso que la antropología es una disciplina con un origen colonial explícito que sirvió, principalmente, a la expansión del imperio británico. Sin embargo, desde mediados del siglo XX hasta la actualidad esta disciplina ha tenido una posición fuertemente crítica hacia esas prácticas coloniales, reinventándose así por medio de nuevas maneras de hacer etnografía que ponen sus herramientas y conocimientos a disposición de las voces que han sido invisibilizadas. Aunque desde la antropología aún se continúan reali- 
zando investigaciones relacionadas con el mundo militar (Rey, 2021), el uso de la disciplina para la lucha contrainsurgente es muy cuestionado.

$\mathrm{Si}$ bien uno de los elementos centrales de la lucha contrainsurgente es el entendimiento de la cultura, la instrumentalización de esta para crear "enemigos" a través de generalizaciones puede ser bastante peligrosa (Castaño, 2010). El Hтs tenía en sus filas a científicos sociales, que además no eran expertos sobre los lugares donde eran desplegados, y aun así tenían la misión de observar prácticas culturales y roles sociales para identificar posibles amenazas (González, 2010).

El uso de las habilidades antropológicas con el fin de categorizar, en medio de una zona de guerra, como amenazas a individuos o grupos se convirtió en una práctica condenada por la Asociación Americana de Antropología, máximo ente que regula la disciplina en ese país. El HTS se volvió una práctica no grata para la comunidad académica y, de la misma manera, varios antropólogos que fueron actores activos en estas operaciones tuvieron posiciones críticas a su salida. El poner la etnografía a disposición de prácticas "securitarias" (Buzan et al., 1998) se convirtió en motivo de enormes tensiones que llevaron a que el programa perdiera popularidad también ante la opinión pública.

Teniendo en cuenta esto, la AIN, como se ha visto, es una estrategia que se reconoce como psicológica y de apoyo a las operaciones militares tradicionales, además tiene un fuerte enfoque en las acciones cívico-militares y hace énfasis en las relaciones entre el personal de la Ain y las poblaciones con el fin de obtener la información. Tanto el HTs como la AIN se plantearon la necesidad de tener una visión holística de los espacios donde operan las insurgencias; el primero fue más allá y llevó personal que se había preparado profesionalmente para mirar el mundo de esa manera, mientras que el segundo continúa realizando estas prácticas a través de una variedad de personal civil y uniformado.

Si bien, en general, la antropología se ha alejado de la lucha contrainsurgente, ya que éticamente no se considera apropiado poner el conocimiento al servicio de estas prácticas militares (Whitehead, 2009) que transformaron a la antropología en arma (Sluka, 2010), es evidente que las operaciones psicológicas se continuarán realizando a través del uso de conocimientos y herramientas etnográficas. Por esta razón, y teniendo en cuenta las críticas que se 
le hicieron al HTs, la AIN debe ser una práctica consciente de las lecciones aprendidas de la experiencia norteamericana y su relación con las ciencias sociales. Dado que la AIN emplea conocimientos y metodologías de las ciencias sociales, tiene la responsabilidad de ser igualmente crítica como lo son las disciplinas de las que se apropia. En tal sentido, esta estrategia de las Fuerzas Militares colombianas no debe desconocer lo problemático que ha sido, a través de la historia del conflicto armado en el país, la construcción de la categoría de "enemigo" (Castaño, 2010). Más aún si esa alteridad que se califica como amenaza se ha construido mediada por el miedo, el desconocimiento y los prejuicios. Cuando se trabaja de la mano con poblaciones que han sido vulneradas sistemáticamente y que se encuentran en procesos de transición (Castillejo-Cuéllar, 2014) es necesario trabajar desde la empatía.

El uso de conocimientos etnográficos con el fin de obtener información pone en riesgo a las poblaciones (Castaño, 2010) y si bien la población civil es un actor importante a la hora de combatir la insurgencia, estas prácticas se deben llevar a cabo con la misma rigurosidad, responsabilidad y cuidado para dejar a un lado las generalizaciones que pueden llevar a extremos peligrosos al momento de construir la alteridad como una amenaza. Aunque la ArN contempla los Derechos Humanos como pilar de la estrategia, es necesario ir más allá al considerar los dilemas éticos que se presentan en el área. Como consecuencia, el manejo responsable de la información obtenida se vuelve un imperativo.

\section{Un manejo responsable de la información}

La AIN clasifica como un "ambiente de información" al escenario donde se desarrolla, lo cual muestra dos implicaciones que vale la pena evidenciar. Primero, la construcción de propaganda y contrapropaganda, como ya se expuso al inicio, y, segundo, la utilización de la información obtenida de la población civil. Para los objetivos de este apartado resulta relevante discutir la segunda. La información es uno de los elementos centrales de la investigación antropológica y asimismo se entiende la necesidad de proteger a los "informantes" y de respetar los lazos de confianza creados (Sluka, 2010). En los casos del HTs y la AIN, la información que se obtiene es enviada a los comandantes 
con el fin de que creen estrategias informadas, pero la recopilación de estos datos y su posterior interpretación por un tercero pueden llevar a tomar decisiones descontextualizadas y poco efectivas. Aunque en otros casos un uso responsable de la información puede facilitar procesos. El antropólogo Marcus Griffin, quien fue parte del HTs, escribe en sus notas etnográficas sobre el tema:

Pero lo que es más importante es que el comandante tuvo la información a mano para tomar su decisión y que se le brindó la información sociocultural necesaria, entre otro tipo de información, para tomar su decisión. Es probable que hoy en día haya personas vivas e ilesas, que de otra manera, si el comandante hubiera tomado una decisión diferente basada, quizás, en menos información. (Griffin, 2010, p. 229)

El manejo de la información está mediada por la transparencia y la consciencia de lo que se obtiene. Una de las críticas comunes hacia el HTs se refería a que si bien esta información obtenida, según el gobierno, no era clasificada porque no correspondía a inteligencia, nunca estuvo disponible el tipo de información que fue recolectada ni qué usos se le dieron (González, 2008). La AIN, al plantearse como una estrategia que busca poner soldados en medio de las poblaciones para analizar variables operacionales a través de la información, debe hacer un trabajo exhaustivo en la capacitación de personal para que la obtención y uso de la información sea apropiado y legítimo. Sobre esto es necesario aclarar que se entiende que existen operaciones donde la información es de carácter clasificado, lo cual corresponde a inteligencia militar que tiene otro tipo de implicaciones, mientras que la AIN se plantea como otro tipo de operación que se refiere al reconocimiento integral de poblaciones, información que debe ser accesible para el control y el monitoreo.

Teniendo en cuenta que la AIN es un medio para lo que se denomina la Acción Unificada del Estado, donde se busca que diversas entidades estatales lleguen a territorios donde no han logrado tener presencia, es importante que la información que se entregue a estas instituciones y otras que colaboran como las $\mathrm{ONG}$ - sea legítima, transparente y obtenida de manera ética, para así continuar en una ruta hacia la construcción de paz, la cual incluye a la población civil y las entidades mencionadas anteriormente. 


\section{Dilemas éticos de la práctica}

Este es, sin duda, el punto más álgido del tema. Las opiniones, por parte de los opositores del HTs, fueron contundentes en exponer la incompatibilidad del programa con la ética del trabajo antropológico:

En términos éticos, el HTS da prioridad a los requerimientos militares: soporte en combate y contrainsurgencia, recolección de inteligencia, y tareas eufemísticamente llamadas "fase cuatro" u "operaciones de estabilización". Estos aspectos del HTS parecen violar la ética antropológica, particularmente las "principales obligaciones éticas con las personas con quienes trabajan" los investigadores, y la necesidad de "asegurar que su investigación no atente contra la seguridad, dignidad o privacidad de las personas con quienes trabajan" (American Anthropological Association, 1998). Además, el conocimiento local sobre jerarquías políticas, estructuras de parentesco y redes sociales pueden facilitar un tipo de regla indirecta, como podrían cooptar a los jefes regionales. (González, 2010, p. 236)

Este tipo de afirmaciones respaldadas por la Asociación Americana de Antropología condenaron la relación del HTs con la utilización de prácticas etnográficas y personal entrenado en el tema. La incompatibilidad del quehacer antropológico con su utilización militar en este caso resulta prácticamente un hecho, por la manera en que se formuló el HTs y la forma como fue implementado en terreno. Los dilemas éticos son centrales en una disciplina como la antropología, de forma que no es posible una investigación etnográfica sin una conciencia plena de las limitaciones (Weiss \& Rey, 2020) y las responsabilidades que esto conlleva.

Pero, ¿por qué resulta pertinente la reflexión ética desde la antropología hacia la AIN? Si bien la AIN no es un sistema desarrollado propiamente a partir de la antropología (como sí lo fue el HTS), sus prácticas resultan similares a la investigación etnográfica: el relacionamiento con la población, la visión holística de las situaciones en campo y la construcción de categorías sobre la alteridad, son algunos ejemplos. Esto quiere decir que la AIN tiene un nivel de responsabilidad sobre el desarrollo de estas prácticas y debe aprender de la experiencia del HTs para no cometer los mismos errores que llevaron a la eliminación de la propuesta en el año 2014. 
La AIN es una estrategia que se proyecta mucho más allá de la lucha contrainsurgente — como se verá en el siguiente acápite—, por eso su práctica debe ser coherente en términos éticos, como lo demuestra el desarrollo del trabajo etnográfico realizado por ańos y sus resultados: "El poder de la etnografía [...] ilumina las complejidades que subyacen al orden y desorden contemporáneos a escala nacional y mundial” (Kelly et al., 2010, p. 3). La AIN tiene un potencial único para la construcción de paz en la actualidad, a través de sus programas y en conjunto con las diversas entidades. Aunque se reconoce que el escenario global está en constante cambio y con este se transforman las tareas que se deben desarrollar, es necesario que este tipo de programas mantengan y fortalezcan una ética que permita continuar con las funciones de la AIN y afrontar los retos que plantean las nuevas nociones de la seguridad a las Fuerzas Militares.

\section{Nuevos factores de transformación: Acción Integral y construcción de paz}

\section{Seguridad multidimensional}

En el marco de las nuevas nociones de seguridad, es necesario partir del contexto de la posguerra fría, donde las guerras dejaron de ser internacionales y se transformaron en conflictos internos de Estados. Además, se debe tener en cuenta que el fenómeno de la globalización generó una nueva demanda de seguridad que no podía ser atendida exclusivamente por las fuerzas militares. En ese sentido, la Unión Europea decidió lanzar en el año 2002 la Estrategia Europea de Seguridad, donde define los principales desafíos mundiales: además de las guerras existentes, se contemplan los desplazamientos forzados, la pobreza, la aparición de nuevas enfermedades, el fracaso económico de los Estados y la escasez de recursos naturales. En este sentido, se genera una nueva visión más amplia acerca de la seguridad: la seguridad multidimensional, que incluye la cooperación y el desarrollo, y donde se reconoce que estas amenazas requieren de distintos instrumentos, como servicios militares, judiciales, políticos y humanitarios para hacerles frente (Font \& Ortega, 2012). 
Partiendo de este nuevo concepto de seguridad multidimensional en el sistema internacional, la Organización de Estados Americanos presentó en el año 2003 la Declaración sobre Seguridad en las Américas, aprobada por todos los Estados — con lo cual le confiere un valor jurídico superior-, en la cual se establece que la aparición de nuevas amenazas, además de las tradicionales, representa desafíos desconocidos en términos de seguridad en el hemisferio (Blackwell, 2015). En este nuevo marco de acción se reconoce que los nuevos desafíos son problemas intersectoriales, lo cual significa que requiere de la respuesta de las distintas organizaciones gubernamentales, las Fuerzas Militares, el sector privado, la sociedad civil y las organizaciones internacionales (Organización de Estados Americanos, 2003).

Además, el Programa de Naciones Unidas para el Desarrollo (PNUD) desarrolló el concepto de seguridad humana, en el cual los principales factores de la seguridad nacional, la seguridad territorial y los armamentos se transforman en dos componentes más amplios, la seguridad de la población y la seguridad basada en el desarrollo humano sostenible. De igual manera, el PNUD establece siete categorías sobre las cuales se basa la seguridad humana: seguridad económica, seguridad alimentaria, seguridad en materia de salud, seguridad ambiental, seguridad personal, seguridad de la comunidad y seguridad política (Castillo \& Niño, 2018).

El nuevo enfoque multidimensional y humano de la seguridad que se observa en el ámbito internacional hace que Colombia actualice su doctrina, de modo que se observa un cambio en las Fuerzas Militares para convertirse en agentes dinamizadoras del fortalecimiento territorial para fomentar el desarrollo económico, social y la protección del medio ambiente y los recursos naturales a largo plazo.

\section{Nuevos roles de las Fuerzas Militares}

Partiendo del contexto político expuesto al inicio, es necesario cuestionar los nuevos roles que tendrán las Fuerzas Militares para desarrollar su misión en el marco del posacuerdo, alejándose del concepto de seguridad nacional y acercándose al de seguridad multidimensional. Este último se puede enmarcar en el contexto de las operaciones "más allá de la guerra", en las cuales las 
Fuerzas Militares asisten emergencias por desastres naturales, entregan suministros básicos a población vulnerable y brindan apoyo tras el fin de conflictos armados. En el caso de Colombia, es necesario tener en cuenta la proyección del Plan Estratégico Militar 2030, que se establece a partir de un ejercicio prospectivo realizado por el Ministerio de Defensa y la Policía Nacional, en el cual se decreta la consolidación de las Fuerzas Militares para la finalización del conflicto, la reducción de los grupos al margen de la ley y el crimen organizado transnacional, así como la diplomacia para la seguridad a partir de la cooperación con otros países, buscando resguardar los esfuerzos desarrollados en el marco de la implementación de los acuerdos de paz (Torrijos et al., 2018).

Debido al nuevo marco internacional, la reconfiguración de las nuevas amenazas exige al Ministerio de Defensa Nacional definir la forma en la que se deben modernizar y fortalecer las Fuerzas Militares. Actualmente, esto se desarrolla por medio del documento maestro Transformación y futuro de la Fuerza Pública - 2030, donde los nuevos roles de las Fuerzas Militares se configuran a partir de siete áreas misionales, que se denominan funcionales y no tradicionales. En las funcionales se encuentran: la convivencia y la seguridad ciudadana, y la seguridad pública y la Defensa Nacional; en las no tradicionales se encuentran: la gestión de riesgo, la cooperación internacional, el desarrollo del país y la protección del medioambiente y de los recursos naturales (Ministerio de Defensa Nacional, 2010).

Dicho documento se realiza con base en la doctrina de AIN y a través de la conformación de los Comités de Revisión Estratégica e Innovación de Acción Integral (CREI), los cuales iniciaron en el 2011 y se materializaron en un conjunto de innovaciones para la construcción del Plan de Campaña Espada de Honor en el 2012. Estos comités reúnen los esfuerzos de las Fuerzas Militares junto con la Policía Judicial para la reducción de actores y actividades ilegales en regiones como Córdoba, Bajo Cauca, Antioquia, Chocó, Guaviare, Vaupés, Cundinamarca, Meta, Amazonas, Putumayo y Caquetá, donde se requiere realizar acciones interagenciales y unificadas (Ministerio de Defensa Nacional, 2010).

En el marco del proceso de paz, el plan Espada de Honor facilitó al gobierno tener un escenario político para desarrollar las conversaciones con las 
FARC y darle paso al Plan Estratégico Militar de Estabilización y Consolidación Victoria en el 2017 para la protección institucional y territorial en el marco del posacuerdo, que se consolida con el plan Victoria Plus debido a los nuevos fenómenos de criminalidad para la búsqueda total de la paz en Colombia (Comando General de las Fuerzas Militares, 2018).

\section{Consolidación territorial: herramienta de los gobiernos para el posconflicto}

Ahora bien, en el establecimiento de la doctrina de AIN para la consolidación del posconflicto existe un aspecto fundamental, el cual tiene como objetivo la recuperación de los territorios y la búsqueda de legitimidad y gobernabilidad en las zonas donde hay ausencia del Estado. Desde esta perspectiva, los municipios y los departamentos se convierten en las entidades más importantes para la resolución de problemas, pues se encuentran en el nivel más cercano a la población y se relacionan con las primeras acciones que debían realizar las Fuerzas Militares para buscar el bienestar de la población después de las operaciones contra las FARC, como se observa en el Plan Espada de Honor (Mejía, 2015).

La idea de que parte considerable de los recursos que conforman los planes estratégicos de las Fuerzas Militares se deban destinar a solucionar necesidades básicas de la población más vulnerable en el marco del conflicto en aspectos como educación, salud, servicios públicos, necesidades básicas, entre otras, adquiere gran importancia debido a que se soporta en los conceptos de gobernanza y gobernabilidad, ya que la única manera en la que se da la verdadera derrota de los grupos al margen de la ley es desarrollando un trabajo sincronizado de las Fuerzas Militares junto con el gobierno nacional. Es decir, la estrategia consiste en que las Fuerzas Militares recuperen el territorio estratégica y militarmente para que el gobierno pueda hacer presencia con sus instituciones municipales para desarrollar las actividades propias del Estado (Mejía, 2015).

Este plan se estableció en el 2014 durante el gobierno Santos con la creación de la Política Nacional de Consolidación y Reconstrucción Territorial (PNCRT), como política gubernamental que sustenta la doctrina para la protec- 
ción del territorio en las zonas más neurálgicas del país (Comando General de las Fuerzas Militares, 2017).

\section{Aplicación de la Acción Integral en Colombia}

En la actualidad, otra forma en la que se observa la aplicación de la doctrina de AIN es a partir del desarrollo del programa Fe en Colombia, que busca la articulación de las instituciones gubernamentales, el sector privado y la cooperación internacional para mejorar las condiciones de vida de la población más vulnerable del territorio nacional dentro del marco de la seguridad multidimensional (Ejército Nacional de Colombia, 2016). Este nuevo proyecto nace de las necesidades que se generan en zonas sin protección estatal y busca una política pública integral que cobije a todo el territorio nacional.

Actualmente, las Fuerzas Militares cuentan con las capacidades necesarias para desarrollar la doctrina de AIN y evidencian ventajas frente a otras instituciones públicas y privadas cuando se despliegan en el territorio nacional. Por lo tanto, la articulación institucional está enfocada en el programa y recurre a las capacidades de las Fuerzas Militares para apoyar la AIN. Esto permite aprovechar un potencial específico para fomentar el desarrollo económico y social en las zonas del suroccidente colombiano — que afrontan nuevas problemáticas provenientes del conflicto con actores al margen de la ley- y establecer líneas de acción con enfoque comunitario relacionadas con asuntos étnicos, víctimas, madres cabeza de hogar, violencia de género, comunidad LGBTIQ y comunidades religiosas (Comando General de las Fuerzas Militares, 2017).

\section{Conclusiones}

Las Operaciones Militares Distintas a la Guerra han respondido en los últimos años como un modo de combate efectivo, de manera que han sido utilizadas en la gran mayoría de escenarios de guerra que se viven en la actualidad, pero que, a pesar de esto, habían sido poco consideradas antes de la Guerra Fría. Por lo tanto, gracias a hechos como la Segunda Guerra Mundial y la Guerra de Vietnam, los Estados Unidos de América y sus aliados han podido 
encontrar la forma de paliar las ventajas que habían adquirido los nuevos actores de los conflictos armados y sus métodos de convencimiento hacia la población civil, ya fuera mediante el uso de propaganda de guerra, o mediante las relaciones de cercanía que mantenían con la población. En el caso concreto de Colombia, habiendo vivido un extenso conflicto armado, se ha comprendido por experiencia propia y ajena que la población civil es necesaria para la victoria en los nuevos campos de batalla, lo cual ha dado paso a la estrategia de AIN, que se creó en el país desde los años sesenta.

Sin embargo, existe una gran responsabilidad institucional al momento de crear lazos con la comunidad, especialmente en cuanto a temas éticos. $\mathrm{Al}$ examinar los errores cometidos por el HTs se genera una serie de elementos que la AIN debería observar para que continúe construyendo prácticas éticas en torno a la información que se obtiene mediante las operaciones psicológicas que realiza. Además, como estas operaciones tienen una similitud con el trabajo etnográfico, es importante que se haga una capacitación rigurosa al personal que ejecuta este tipo de acciones, para no poner en riesgo la vida de la población civil a la que se están acercando. Si se realiza un uso ético, transparente y responsable de las operaciones psicológicas de la AIN, se puede cumplir el objetivo de la estrategia, que va más allá de la recopilación de información. Contar con unas bases éticas sólidas le permite a la AIN enfrentarse a los retos que tiene en la actualidad.

La globalización es el contexto fundamental para mantener actualizada la doctrina de AIN. Como se puede observar, la seguridad es un fenómeno cambiante que es permeado por la realidad que enfrenta cada Estado, de manera que es necesario hacer un análisis de la realidad internacional periódicamente para que la doctrina se actualice constantemente y así pueda responder a las necesidades de la sociedad colombiana. La doctrina de AIN, como mecanismo de construcción de paz, se desarrolla actualmente por medio del Plan Estratégico Militar de Estabilización y Consolidación Victoria, la Política Nacional de Consolidación y Reconstrucción Territorial y el instructivo Fe en Colombia, donde se consolidan la protección del territorio, la reducción de grupos al margen de la ley y el desarrollo económico y social. Por lo tanto, se puede afirmar que es una herramienta efectiva que se encarga de estudiar las 
zonas históricamente golpeadas por el conflicto para consolidar los territorios y, de esta manera, hacer posible la construcción de legitimidad y gobernabilidad del Estado.

\section{Referencias}

Bados, V. M., \& Durán, M. (2015). Las “nuevas guerras”: Una propuesta metodológica para su análisis. Revista UNISCI, (38), 9-33. https://doi.org/10.5209/rev_runi.2015.n38.49643

Blackwell, A. (2015). Seguridad multidimensional: "Enfrentando nuevas amenazas". En Seguridad, ciencia y defensa (pp. 153-158). Organización de Estados Americanos.

Buzan, B., \& Wæver, O., \& Wilde, J. (1998). Security. A new framework for analysis. Lynne Rienner Publishers.

Castaño, P. (2010). The categorization of people as targets of violence: A perspective on the Colombian Armed Conflict. En J. Kelly, B. Jauregui, S. Mitchell \& J. Walton (eds.), Anthropology and global counterinsurgency (pp. 53-66). University of Chicago Press.

Castillejo-Cuéllar, A. (2014). La localización del daño. Etnografía, espacio y confesión en el escenario transicional colombiano. Horizontes Antropológicos, (42), 213-236. https:// doi.org/10.1590/s0104-71832014000200009

Castillo Castañeda, A., \& Niño González, C. A. (2018). La doctrina de Acción Integral como política de seguridad en el posconflicto armado en Colombia. En C. A. Niño (comp.), Perspectivas y prospectivas de la seguridad en Colombia (pp. 121-148). USTA. https:// doi.org/10.2307/j.ctvb9391v.10

Clausewitz, C. (1999). De la guerra. Editorial Idea Universitaria.

Comando General de las Fuerzas Militares. (2017). Manual de acción integral conjunta. Fuerzas Militares de Colombia.

Comando General de las Fuerzas Militares. (2018, agosto 1). De Espada de Honor a Victoria Plus. Las Fuerzas, (51), 1-3. https://www.cgfm.mil.co/sites/default/files/glazed-cmsmedia/las_fuerzas_ed_51.pdf

Cruz Rodríguez, E. (2015). Relaciones cívico-militares, negociaciones de paz y postconflicto en Colombia. Criterio Jurídico Garantista, 8(13), 12-41. https://doi. org/10.26564/21453381.581

Ejército Nacional de Colombia. (2016). Fe en Colombia: un programa de todos para todos. Ejército Nacional de Colombia.

Font, T., \& Ortega, P. (2012). Seguridad nacional, seguridad multidimensional, seguridad humana. Papeles de Relaciones Ecosociales y Cambio Global, (119), 161-172. https:// www.fuhem.es/papeles_articulo/seguridad-nacional-seguridad-multidimensionalseguridad-humanal

Goldstein, F., \& Jacobowitz, D. (1996). Psycological operations: An introduction. En F. Goldstein \& D. Jacobowitz (Eds.), Psycological operations. Principles and cases studies 
(pp. 5-16). Air University Press. https://media.defense.gov/2017/Apr/07/2001728209/1/-1/0/B_0018_GOLDSTEIN_FINDLEY_PSYCHLOGICAL_OPERATIONS.PDF

González, R. J. (2008). 'Human terrain' Past, present and future applications. Anthropology Today, 24(1), 21-26. https://doi.org/10.1111/j.1467-8322.2008.00561.x

González, R. J. (2010). Indirect rule and Embedded Anthropology: Practical, theoretical, and ethical concerns En J. Kelly, B. Jauregui, S. Mitchell \& J. Walton (eds.), Anthropology and global counterinsurgency (pp. 231-244). University of Chicago Press.

Griffin, M. (2010). An Anthropologist among the Soldiers: Notes from the Field. En J. Kelly, B. Jauregui, S. Mitchell \& J. Walton (eds.), Anthropology and global counterinsurgency (pp. 215-230). University of Chicago Press.

Hoffman, F. (2007). Conflict in the 21st Century: The rise of Hybrid Wars. Potomac Institute for Policy Studies.

Illera, O., \& Ruiz, J. C. (2018). Entre la política y la paz: Las Fuerzas Militares tras la firma del Acuerdo de Paz. Araucaria. Revista Iberoamericana de Filosofía, Política y Humanidades, 20(39), 509-533. https://doi.org/10.12795/araucaria.2018.i39.24

Kaldor, M. (2012). New \& old wars: Organized violence in a global era. Polity Press. Cambridge.

Kelly, J., \& Jauregui, B., \& Mitchell, S., \& Walton, J. (2010). Introduction: Culture, counterinsurgency, conscience. En J. Kelly, B. Jauregui, S. Mitchell \& J. Walton (eds.), Anthropology and global counterinsurgency (pp. 1-16). University of Chicago Press.

La paz híbrida de Mary Kaldor. (2017, octubre 10). El Espectador. https://www.elespectador. com/colombia2020/pedagogia/la-paz-hibrida-de-mary-kaldor-articulo-855885

Lind, W., \& Thiele, G. (2015). 4th Generation Warfare Handbook. Castalia House.

Leal Buitrago, F. (1994). El oficio de la guerra: La seguridad nacional en Colombia. IEPRI.

McFate, M. (2005). The military utility of understanding adversary culture. Joint Force Quarterly, (38), 42-48.

McFate, M., \& Jackson, A. V. (2006). The object beyond war: Counterinsurgency and the four tools of political competition. Military Review, 13-26. https://apps.dtic.mil/dtic/tr/fulltext/u2/a489124.pdf

Mejía Quintero, O. A. (2015). La acción integral: Herramienta de gestión de los gobiernos municipales focalizados por la Política Nacional de Consolidación y Reconstrucción Territorial. Equidad \& Desarrollo, (23), 127-145. https://doi.org/10.19052/ed.3430

Ministerio de Defensa Nacional. (2010). Transformación y futuro de la Fuerza Pública. Ministerio de Defensa.

Montoya, M. (2007). La Acción Integral: una estrategia para ganar la guerra. Estudios en Seguridad y Defensa, 2(3), 18-24. https://doi.org/10.25062/1900-8325.151

Moreno Mancera, J. D. (2014). Relaciones cívico-militares en Colombia: Supremacía y control de los partidos políticos sobre la organización militar. Revista Científica General José María Córdova, 12(13), 332-352. https://doi.org/10.21830/19006586.166 
Niño, C., \& Castillo, A. (2018). La Doctrina de Acción Integral como política de seguridad en el posconflicto armado en Colombia. En C. Niño (comp.), Perspectivas y prospectivas de la seguridad en Colombia (pp. 121-148). Universidad Santo Tomás. https:// doi.org/10.2307/j.ctvb9391v.10

Organización de Estados Americanos. (2003). Declaración sobre seguridad en las Américas. http://www.oas.org/juridico/spanish/decl_security_sp.pdf

Ortega, R. (2011). La guerra asimétrica y las operaciones de información. Military Review, 21-28. https://www.armyupress.army.mil/Portals/7/military-review/Archives/Spanish/ MilitaryReview_20110630_art006SPA.pdf

Paret, P. (1992). Creadores de la estrategia moderna desde Maquiavelo hasta la era atómica. Ministerio de Defensa de España.

Petraeus, D. (2006). Learning counterinsurgency: Observations from soldiering in Iraq. Military Review, (January-February), 45-55. https://www.armyupress.army.mil/Portals/7/ military-review/Archives/English/MilitaryReview_2006CR1031_art010.pdf

Rey Pinto, E. M. (2021). En las aguas de la distinción: imaginarios sobre el prestigio en la Armada Nacional de Colombia. Revista Cientifica General José María Córdova, 19(35), 625-642. http://dx.doi.org/10.21830/19006586.783

Rodríguez Morales, L. F. (2014). Los pilares de la politica integral de seguridad y defensa para la prosperidad como elementos determinadores para su consolidación. [Tesis de especialización, Universidad Militar Nueva Granada]. Repositorio UMNG. https://repository.unimilitar.edu.co/bitstream/handle/10654/12286/LOS\%20PILARES\%20DE\%20 LA $\% 20$ POL $\%$ c3\%8dTICA\%20INTEGRAL \%20DE\%20SEGURIDAD\%20Y\%20 DEFENSA\%20PARA\%20LA\%20PROSPERIDAD.pdf? sequence=1 \&isAllowed=y

Ruiz Mora, J. A. (2007). Acción integral, hacia una doctrina unificada. Estudios en Seguridad y Defensa, 2(3), 26-32. https://doi.org/10.25062/1900-8325.152

Silva Urbano, M. G. (2014). La acción integral como una estrategia efectiva hacia la consolidación de la seguridad y la defensa nacional [Tesis de especialización, Universidad Militar Nueva Granada]. Repositorio UMNG. https://repository.unimilitar.edu. co/bitstream/handle/10654/13730/LA\%20ACCI\%c3\%93N\%20INTEGRAL\%20 COMO\%20UNA\%20ESTRATEGIA\%20EFECTIVA\%20HACIA\%20LA\%20 CONSOLIDACI\%c3\%93N\%20DE\%20LA\%20SEGURIDAD\%20Y\%20LA\%20 DEFENSA\%20NACIONAL\%20CON\%20COME.pdf? sequence=2\&isAllowed=y

Sluka, J. A. (2010). Curiouser and curiouser: Montgomery McFate's strange interpretation of the relationship between anthropology and counterinsurgency. PoLar: Political and Legal Anthropology Review, (33), 99-115. https://doi.org/10.1111/j.1555-2934.2010.01068.x

Sun Tzu. (1999). El arte de la guerra. Editorial Panamericana.

Torrijos Rivera, V., Balaguera Sarmiento, L. F., \& Ardila Castro, C. A. (2018). Visión desde Colombia. Roles de la Fuerza Pública colombiana en escenarios de posconflicto. Cuadernos de Estrategia, (195), 13-37. https://dialnet.unirioja.es/servlet/articulo?codigo=6532151 
Valencia, A. (1959). Defensa nacional y guerra revolucionaria. Revista Fuerzas Armadas, (24), 393-399.

Valencia, A. (2006). Teoría y práctica de la acción integral. Revista Fuerzas Armadas, (198), 6-11.

Weiss, C., \& Rey Pinto, E. M. (2020). Twenty years after UNSCR 1325: Equal access to military education in the Western Hemisphere? Estudios en Seguridad y Defensa, 15(30), 303-325. https://doi.org/10.25062/1900-8325.280

Whitehead, N. L. (2009). Ethnography, torture and the Human Terrain/Terror System. Fast Capitalism, 5(2). https://fastcapitalism.uta.edu/5_2/Whitehead5_2.html 